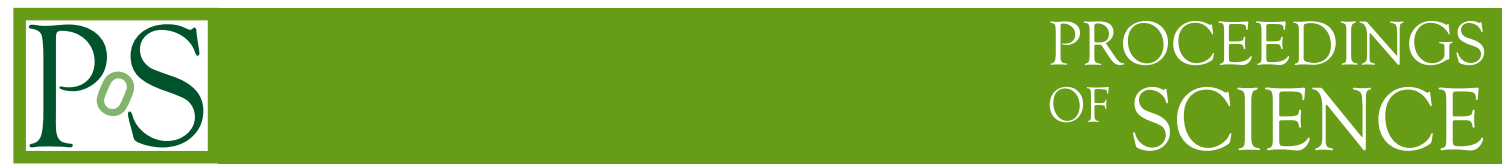

\title{
Maser observation in VY CMa with VERA
}

\author{
Yoon Kyung Choi* \\ Department of Astronomy, Graduate School of Science, The University of Tokyo, Tokyo \\ 113-0033, Japan \\ E-mail: yoonkyung.choi@nao.ac.jp
}

\begin{abstract}
We present the results of multi-epoch VERA (VLBI Exploration of Radio Astrometry) observations of $\mathrm{H}_{2} \mathrm{O}$ masers at $22 \mathrm{GHz}$ and ${ }^{28} \mathrm{SiO}$ masers at $43 \mathrm{GHz}$ in the supergiant VY Canis Majoris (hereafter, VY CMa). We estimate the inner motion of $\mathrm{H}_{2} \mathrm{O}$ masers over 6 months and that of $\mathrm{SiO}$ masers over 1 month. Using the inner motion, we calculated the statistical parallax of VY CMa. The size of the emitting region for ${ }^{28} \mathrm{SiO}$ masers is $\mathrm{R}_{\mathrm{SiO}} \sim 1.81-2.89 \mathrm{R}_{*}$ and it is consistent with the previous study.
\end{abstract}

The 8th European VLBI Network Symposium

September 26-29, 2006

Toruń, Poland

${ }^{*}$ Speaker. 


\section{Introduction}

Red supergiants have a high mass-loss rate and are often surrounded by shells of gas and dust. $\mathrm{H}_{2} \mathrm{O}$ and $\mathrm{SiO}$ masers are found in the circumstellar envelopes (CSE) of supergiants. These masers are an important tool to study the structures and the dynamics of the CSE, the mass-loss processes, and the evolutions of these type of stars. VY CMa, the star we have observed, is one of the brightest supergiants in the Galaxy. $\mathrm{OH}, \mathrm{H}_{2} \mathrm{O}$ and $\mathrm{SiO}$ masers are found in its CSE. The luminosity of VY $\mathrm{CMa}$ is $5.5 \times 10^{5} \mathrm{~L} \odot$ and the distance is $1.5 \mathrm{kpc}$ [1].

\section{Observations}

We observed $\mathrm{H}_{2} \mathrm{O}$ masers at $22 \mathrm{GHz}$ and ${ }^{28} \mathrm{SiO}$ masers at $43 \mathrm{GHz}$ in the supergiant VY CMa using VERA (VLBI Exploration of Radio Astrometry). $\mathrm{H}_{2} \mathrm{O}$ maser observations were conducted over 3 epochs in May 2003, October 2003, and December 2003 with the restoring beam of $2.1 \times$ 0.8 mas. ${ }^{28} \mathrm{SiO}$ maser observations were carried out in April 2006 and May 2006 with the restoring beam of $1.0 \times 0.4$ mas.

\section{Results and Discussion}

The distribution and movements of $\mathrm{H}_{2} \mathrm{O}$ maser features in VY CMa are shown in Fig. 1. To align the maps over three epochs, we assumed that the position of the brightest maser spot at each epoch is the same. In order to investigate the inner motion, maser components which are detected at all three epochs have been chosen. For the chosen components, we estimated the inner motion during six months. The movements of these components are also shown in Fig. 1. Based on the inner motion, we estimated the statistical parallax. The statistical parallax method assumes that the standard deviation of both radial and transverse velocities are identical. The distance to VY CMa estimated using this method is $1.88 \mathrm{kpc}$.
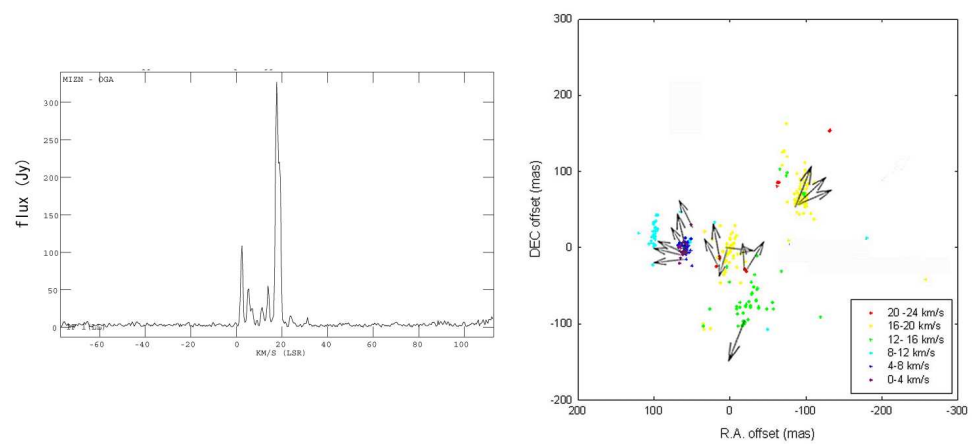

Figure 1: (left) The cross-correlated spectrum of $\mathrm{H}_{2} \mathrm{O}$ masers in VY CMa. (right) The locations and the movements of $\mathrm{H}_{2} \mathrm{O}$ maser features in VY CMa over six months. The arrows on the map indicate the directions of the inner motion.

The distributions of $J=1 \rightarrow 0 v=1$ and $v=2{ }^{28} \mathrm{SiO}$ maser features in VY CMa are shown in Fig. 2. For $J=1 \rightarrow 0 v=1{ }^{28} \mathrm{SiO}$ masers, we estimate preliminary inner motions over one month 
(Fig. 3). The distance to VY CMa based on statistical parallax is $1.18 \mathrm{kpc}$. This result is smaller than that given in the previous study (1.5 kpc, [1]). Compared with the one calculated from the statistical parallax of $\mathrm{H}_{2} \mathrm{O}$ masers, this distance is also underestimated.
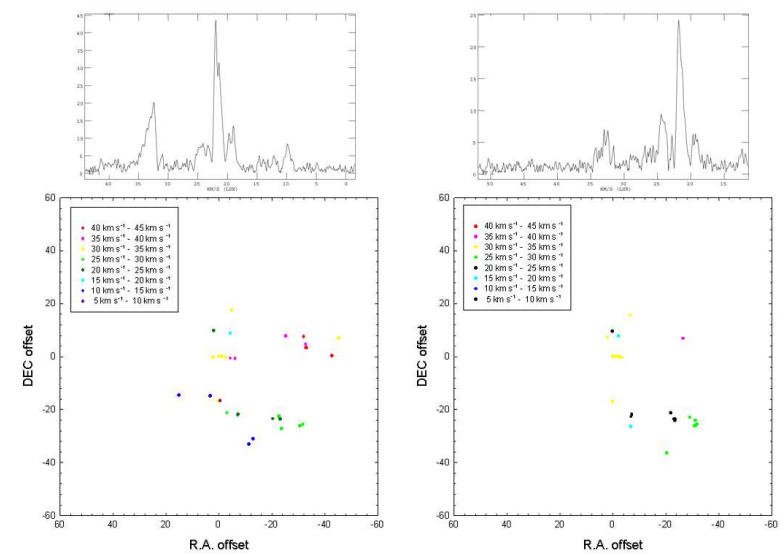

Figure 2: (left) The cross-correlated spectrum and the maser features distribution of $J=1 \rightarrow 0 v=1$ ${ }^{28} \mathrm{SiO}$ masers in VY CMa. (right) The cross-correlated spectrum and the maser features distribution of $J=1 \rightarrow 0 v=2{ }^{28} \mathrm{SiO}$ masers in VY CMa.

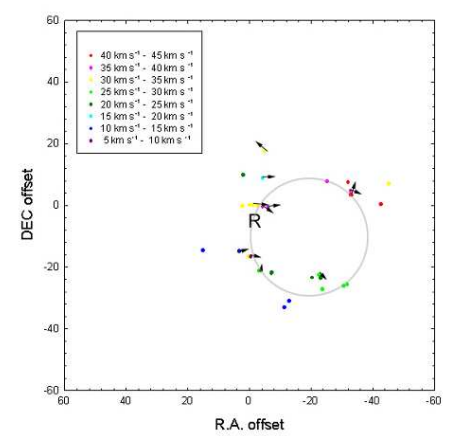

Figure 3: The preliminary inner motions of $J=1 \rightarrow 0 v=1{ }^{28} \mathrm{SiO}$ maser features. $\mathrm{R}$ is the position of $\mathrm{V}_{\mathrm{LSR}}$ $=22 \mathrm{~km} \mathrm{~s}^{-1}$ which is used to align the positions of two epochs.

The adopted radius of the model for VY CMa's circumstellar geometry is $\mathrm{R}_{*}=13 \mathrm{AU}$ [2], and it is 6.91 mas at $1.88 \mathrm{kpc}$ and 11.01 mas at $1.18 \mathrm{kpc}$. The distribution of ${ }^{28} \mathrm{SiO}$ maser features is a ring as shown in Fig. 3, and its diameter is approximately 40 mas. From this we obtain $\mathrm{R}_{\mathrm{SiO}} \sim$ $1.81-2.89 \mathrm{R}_{*}$ and this result is consistent with the notion that $\mathrm{SiO}$ maser are located at typical distances of 2-4 stellar radii from Mira variables and supergiants [3].

\section{References}

[1] C.J. Lada and M.J. Reid, 1978, ApJ, 219, 95

[2] N. Smith, R.M. Humphreys, K. Davidson, R.D. Gehrz, M.T. Schuster, and J. Krautter, 2001, AJ, 121, 1111

[3] P.J. Diamond, A.J. Kemball, W. Junor, A. Zensus, J. Benson, and V. Dhawan, 1994, ApJ, 430, L61 